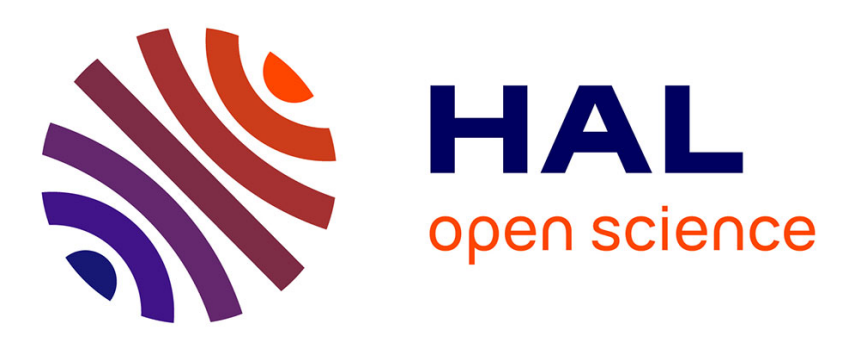

\title{
Thermodynamics of Alternating Quantum-Classical Spin Chains Showing Isotropic Nearest Neighbor Couplings
}

\author{
Jacques Curély, Roland Georges
}

\section{To cite this version:}

Jacques Curély, Roland Georges. Thermodynamics of Alternating Quantum-Classical Spin Chains Showing Isotropic Nearest Neighbor Couplings. Journal de Physique I, 1995, 5 (4), pp.485-499. 10.1051/jp1:1995142 . jpa-00247074

\section{HAL Id: jpa-00247074 https://hal.science/jpa-00247074}

Submitted on 1 Jan 1995

HAL is a multi-disciplinary open access archive for the deposit and dissemination of scientific research documents, whether they are published or not. The documents may come from teaching and research institutions in France or abroad, or from public or private research centers.
L'archive ouverte pluridisciplinaire HAL, est destinée au dépôt et à la diffusion de documents scientifiques de niveau recherche, publiés ou non, émanant des établissements d'enseignement et de recherche français ou étrangers, des laboratoires publics ou privés. 
Classification

Physics Abstracts

$75.50 \mathrm{G}-75.10 \mathrm{D}-75.10 \mathrm{H}$

\title{
Thermodynamics of Alternating Quantum-Classical Spin Chains Showing Isotropic Nearest Neighbor Couplings
}

\author{
Jacques Curély $\left({ }^{1, *}\right)$ and Roland Georges $\left({ }^{2}\right)$ \\ ( $\left.{ }^{1}\right)$ Laboratoire de Cristallographie et de Physıque Cristalline, Université de Bordeaux I, 351 \\ Cours de la Libération, 33405 Talence Cedex, France \\ (2) Laboratoire de Chimie du Solide, Université de Bordeaux I, 351 Cours de la Libération, \\ 33405 Talence Cedex, France
}

(Recelved 15 November 1994, accepted 19 December 1994)

\begin{abstract}
Résumé. - Un traitement général est proposé pour résoudre le problème des chaînes ferrimagnétiques constituées de deux sous-réseaux $(\mathrm{S}, \mathrm{s})$ et caractérısées par des couplages isotropes entre premers voisins ainsi que deux paramètres d'échange. En conséquence, deux cas physiques untéressants sont examinés : i) les chaînes sont exclusıvement composées de couplages ferromagnétiques ou antiferromagnétiques ; ii) les chaînes présentent une alternance régulière de ces deux types de couplages. Des expressions littérales sont données pour la fonction de partition en champ nul, les corrélations spın-spin ainsi que la susceptibilité. Le comportement basse température de la susceptibilité est étudié au moyen de la longueur de corrélation. En particulier, dans le cas de la compensation des moments magnétiques, ll est montré que ce comportement est principalement décrit par la compétition entre la divergence de la longueur de corrélation et l'annulation du moment magnétique de la cellule élémentaire. Pour finir, nous rappelons un test expérımental quı a initié ce modèle théorıque : 1 concerne le composé $\mathrm{MnCu}(\mathrm{obp})\left(\mathrm{H}_{2} \mathrm{O}\right)_{3} \cdot \mathrm{H}_{2} \mathrm{O}$ [où obp=oxamidobis (propıonato)]
\end{abstract}

\begin{abstract}
We propose a general treatment for solving the case of ferrimagnetic chains made up of two sublattices (S,s) and characterized by isotropic couplings between nearest neighbors, as well as two exchange parameters. Therefore, two cases of physical interest are examined: 1) the chains are exclusıvely composed of ferromagnetic or antiferromagnetic couplings; 11) the chains show a regular alternation of these two types of couplings. Closed-form expressions of the zero-field partition function, the spin-spin correlations, as well as the susceptibility, are given. The low-temperature behavior of the susceptibility is studied by means of the correlation length. In particular, in the magnetic moment compensation, we show that this behavior is mainly described by the competition between the divergence of the correlation length and the evanescence of the magnetic moment per unit cell. Finally we recall an experimental test which has initiated this theoretical model: it concerns the compound $\mathrm{MnCu}(\mathrm{obp})\left(\mathrm{H}_{2} \mathrm{O}\right)_{3} \cdot \mathrm{H}_{2} \mathrm{O}$ [where $\mathrm{obp}=\mathrm{oxamidobis}$ (propionato)].
\end{abstract}

$\left(^{*}\right)$ All correspondence should be addressed to Jacques Curély 


\section{Introduction}

One-dimensional magnetism is a field where theoretical and experimental studies have stimulated each other [1-4]. The reason for this strong interest is that the specific behavior of such 1D materials (notably with an infinite critical-temperature domain) and its ability to sometimes be solved exactly, allow for a possible check of experimental results with the proposed specific models [5-7]. To briefly sum up the history of this field, we can say that, at first, the studied compounds were regular homometallic chains, in which the magnetic centers are equally spaced along the chain [8-10]. Then alternating homometallic chains characterized by two intrachain exchange parameters appeared [11-13]. More recently, the first bimetallic chains were described [14-18] and, because of the new possibilities offered by molecular chemistry engineering, alternating bimetallic chains were synthetized. Among these materials, the socalled ferrimagnetic chains offer many interesting features: their solid-state structure exhibits well-separated magnetic chains along which two cationic species occupy host sites, generally with antiferromagnetic nearest neighbor couplings, thus providing the main basic conditions for ferrimagnetism and considerably increasing the number of nontrivial situations [19-21].

From a theoretical point of view, the necessity of interpreting the experimental magnetic properties of the chains described above begins with the determination of the effective spin Hamiltonian. In a previous paper [22] we have established the general conditions which must be obeyed by the chain Hamiltonian for allowing an analytical recurrent treatment: in particular the basic condition is that all the involved spin operators commute. Then the isotropic or anisotropic nature of coupling with nearest spin neighbors plays an important role. In this paper we exclusively focus on chains showing isotropic couplings. When the chain is only composed of quantum spin moments, the Hamiltonıan always contains non-commutating spin operators; therefore approximate techniques are required: spin-wave theory [23], high-temperature series expansions [24-26], Green's function approaches [27], or numerical extrapolations from exact calculations on finite length chains [28-32]. However, for the purely classical case, the commutation condition is fulfilled. Taking into account this aspect, Fisher has given closedform expressions for the main thermodynamical functions of interest [33] and Stanley has generalized this work to classical spins of arbitrary dimensionality [34]. Following a scheme similar to Fisher's, Seiden [35] has solved the problem of a chain showing an alternation of a quantum spin $s=1 / 2$ with a classical one: the presence of classical spins allows one to separate each unit cell operator $\exp \left(-\beta H_{2}\right)$ and a relevant rotation in the spin space authorizes the calculation of the trace for each operator.

At the end of the eighties we generalized Seiden's model to arbitrary spin quantum numbers $[19,36,37]$ but, so far, only the broad outlines have been presented; more recently, using the same principles, we have proposed a model in which whole quantum subsystems alternate with classical spins [38]. In this paper, in Section 2, we recall the basic principles but now we detail the method. In Section 3 we study the low-temperature behavior of $(\mathbf{S}, \mathbf{s})_{N}$ chains. In particular we examine the cases where the chain is exclusively composed of ferromagnetic or antiferromagnetic couplings or is characterized by the regular alternation of these two types of couplings. We specifically discuss the compensation case (no net moment in the ground state) which also reveals subtle aspects of both short- and long-range orderings. Finally, we recall the interpretation of the experimental results obtained on the compound $\mathrm{MnCu}(\mathrm{obp})\left(\mathrm{H}_{2} \mathrm{O}\right)_{3} \cdot \mathrm{H}_{2} \mathrm{O}$ [where obp=oxamidobis(propionato)] because it has initiated the present theoretical work $[19,37]$. 


\section{General Considerations}

Let us consider a Heisenberg exchange coupling chain submitted to an external magnetic field $\mathbf{B}$ applied along the $z$-axis of quantization (see Fig. 1). Thus for a $(2 N+1)$ spin chain $\mathbf{S}_{0} \mathbf{s}_{0}$ $\mathbf{S}_{\imath} \mathbf{S}_{\imath} . \mathbf{S}_{N} \mathbf{S}_{N}$ the most general Hamiltonian may be written

$$
H=\sum_{\imath=0}^{N-1} H_{\imath}^{\mathrm{ex}}+\sum_{\imath=0}^{N} H_{\imath}^{\mathrm{mag}}
$$

with

$$
\begin{gathered}
H_{\imath}^{\mathrm{ex}}=J \mathcal{S}_{\imath} . \mathrm{s}_{\imath}, \quad \mathcal{S}_{\imath}=(1+\alpha) \mathbf{S}_{\imath}+(1-\alpha) \mathbf{S}_{\imath+1}, \\
H_{\imath}^{\mathrm{mag}}=-\left(G S_{\imath}^{z}+g s_{\imath}^{z}\right) B .
\end{gathered}
$$

$S^{z}$ is the $z$ component of the classical vector operator $\mathbf{S}_{\imath}$ (the spin quantum number $S$ is large enough for $\left[S_{\imath}^{x} S_{\imath}^{y}\right]$ to be negligible compared to $S_{\imath}^{x} S_{\imath}^{y}$-classical spin approximation); $g$ and $G$ are the associated Landé factors: they characterize the magnetic ions of the unit cell. $J$ refers to the exchange interaction between the nearest neighbors only: in our writing $J>0$ denotes an antiferromagnetic coupling. Note also that we shall restrict the discussion to the positive

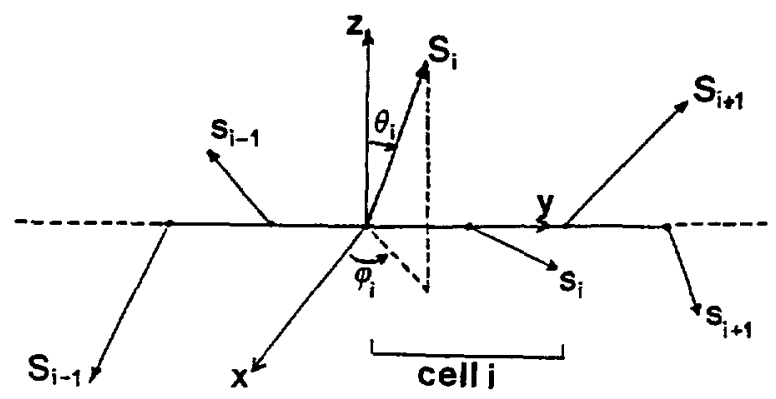

Fig. 1. - Structure of a quantum-classical spin chain.

values of $\alpha$ without loosing generality: this is due to the fact that changing the sign of all the $\alpha$ 's is equivalent to reverse the order of the magnetic ion sequence; consequently the magnetic chain properties are unchanged.

The partition function $Z_{N}(B)$ for the $(2 N+1)$ spin chain may be written

$$
Z_{N}(B)=\int \mathrm{d} S_{0} T r_{\mathbf{s}_{0}} \cdot \int \mathrm{d} S_{\imath} \operatorname{Tr}_{\mathbf{s}_{i}} \cdot \int \mathrm{d} S_{N} T r_{\mathbf{s}_{N}} \exp \left(-\beta\left[\sum_{\imath=0}^{N-1}\left(H_{\imath}^{\mathrm{ex}}+H_{\imath}^{\mathrm{mag}}\right)+H_{N}^{\mathrm{mag}}\right]\right),
$$

where $\operatorname{Tr}_{\mathbf{s}_{\mathbf{t}}}$ represents the trace operation applied to the matrix associated to the spin operator $s_{2}$. The knowledge of the partition function $Z_{N}(B)$ allows one to define the parallel 
magnetization $\mathcal{M}_{N}$ and the zero-field susceptibility $\chi_{N}$ as

$$
\mathcal{M}_{N}=\frac{1}{\beta} \frac{\partial \ln Z_{N}(B)}{\partial B}, \quad \chi_{N}=\frac{1}{\beta Z_{N}(0)}\left(\frac{\partial^{2} Z_{N}(B)}{\partial B^{2}}\right)_{B=0},
$$

where $Z_{N}(0)$ is the partition function expressed for $B=0$. Note that, in order to have a clearer insight, we shall preferably consider $\chi$, the susceptibility referred to the unit cell, and the product $\chi T$ normalized to its infinite temperature value (the Curie constant): $(\chi T)_{n}$.

First we focus on the calculation of the zero-field partition function. Then, in equation (4), the Hamiltonian involved in the exponential argument is reduced to the exchange one $H_{\imath}^{\text {ex}}$; moreover because of the commutativity property of the operators $H_{\imath}^{e x}$ we have

$$
\exp \left(-\beta \sum_{i=0}^{N-1} H_{\imath}^{\mathrm{ex}}\right)=\prod_{\imath=0}^{N-1} \exp \left(-\beta H_{\imath}^{\mathrm{ex}}\right)
$$

Consequently, for each site $i$, we have to evaluate the trace of the operator $\exp \left(-\beta H_{2}^{\mathrm{ex}}\right)$. Using the fact that vector $\mathcal{S}_{\mathrm{i}}$ defined in equation (2) can be chosen as a local axis of quantization we have

$$
\operatorname{Tr}_{\mathbf{s}_{\mathbf{z}}}\left(\exp \left(-\beta H_{\imath}^{\mathrm{ex}}\right)\right)=\sum_{\sigma=-s}^{+s} \exp \left(\sigma \lambda \sqrt{1+\eta \cos \Theta_{\imath, \imath+1}}\right)
$$

with

$$
\lambda=-\beta J \sqrt{2\left(1+\alpha^{2}\right)}, \quad \eta=\frac{1-\alpha^{2}}{1+\alpha^{2}}
$$

(note that, as $\mathbf{S}$ is a unit vector, $S$ has been dropped). As this trace only depends on the angle $\boldsymbol{\Theta}_{\imath, \imath+1}$ between vectors $\mathbf{S}_{\imath}$ and $\mathbf{S}_{\imath+1}$, it becomes possible to expand it on the infinite basis of spherical harmonics

$$
\operatorname{Tr}_{\mathbf{s}_{\imath}}\left(\exp \left(-\beta H_{\imath}^{\mathrm{ex}}\right)\right)=\sum_{\ell=0}^{+\infty} \sum_{m=-\ell}^{+\ell} A_{\ell}(\lambda, \eta, s) Y_{\ell}^{m}\left(\mathbf{S}_{\imath}\right) Y_{\ell}^{-m}\left(\mathbf{S}_{2+1}\right)
$$

with

$$
A_{\ell}(\lambda, \eta, s)=2 \pi \sum_{\sigma=-s}^{+s} \int_{-1}^{+1} \exp (\sigma \lambda \sqrt{1+\eta x}) P_{\ell}(x) \mathrm{d} x
$$

where $P_{\ell}(x)$ is a Legendre polynomial. Then using equation (9) in equation (4) expressed in the zero-field limit as well as the orthogonality condition to which the spherical harmonics obey, the values $m=0$ and $\ell=0$ are selected and we have finally

$$
Z_{N}(0)=4 \pi\left(A_{0}(\lambda, \eta, s)\right)^{N+1}
$$

with

$$
A_{0}(\lambda, \eta, s)=\frac{4 \pi}{\lambda^{2} \eta} \sum_{\sigma=-s}^{+s} \sum_{\varepsilon= \pm 1} \frac{\varepsilon}{\sigma^{2}}\left(v_{\sigma, \varepsilon}-1\right) \exp \left(v_{\sigma, \varepsilon}\right), v_{\sigma, \varepsilon}=\sigma \lambda \sqrt{1+\varepsilon \eta}
$$

At this step it must be noticed that the calculation of $Z_{N}(B)$ involves a further contribution (i.e., the magnetic one described by $H_{\imath}^{\mathrm{mag}}$ ) in each exponential argument: thus the square 
root which appears in equation (7) now contains other terms exclusively depending on $B$ and the $z$ components of $\mathbf{S}_{\imath}$ and $\mathbf{S}_{\imath+1}$ (as the external field is applied along the $z$-axis). Consequently it becomes impossible to expand each trace of $\exp \left(-\beta H_{2}\right)$ as in equation $(9)$ and the magnetization $\mathcal{M}_{N}$ has no closed-form expression.

However, for calculating the susceptibility $\chi$, it is always possible to express it with the help of spin-spin correlations. Considering equations (1)-(5) and using the fact that we deal with an isotropic exchange coupling between nearest spin neighbors, the susceptibility $\chi$ referred to the unit cell can be written as

$$
\begin{gathered}
\chi=\frac{\beta}{3}\left[G^{2}<\left(S_{\imath}^{z}\right)^{2}>+g^{2}<\left(s_{\imath}^{z}\right)^{2}>\right. \\
\left.+\sum_{\jmath \neq \imath}\left(G^{2}<S_{\imath}^{z} S_{\jmath}^{z}>+g^{2}<s_{\imath}^{z} s_{\jmath}^{z}>+G g\left[<S_{\imath}^{z} s_{\jmath}^{z}>+<s_{\imath}^{z} S_{\jmath}^{z}>\right]\right)\right] .
\end{gathered}
$$

The calculation of $\left\langle\left(S_{\imath}^{z}\right)^{2}\right\rangle$ and $\left\langle\left(s_{1}^{z}\right)^{2}\right\rangle$ is obvious; as for the various correlations $\left.<S_{2}^{z} S_{3}^{z}\right\rangle,\left\langle s_{2}^{z} s_{3}^{z}\right\rangle,\left\langle S_{2}^{z} s_{3}^{z}\right\rangle$ and $\left\langle s_{2}^{z} S_{3}^{z}\right\rangle$, the work is similar to that one encountered for evaluating the zero-field partition function $Z_{N}(0)$; it just differs by the introduction of extra terms at rows $i$ and $j$. If $S_{i}^{z}$ is introduced at row $i$ we expand it as

$$
S_{\imath}^{z}=\sqrt{\frac{4 \pi}{3}} Y_{\imath}^{0}\left(S_{\imath}\right)
$$

Then, the orthogonality condition and the specific properties of spherical harmonics allow one to select the values $m=0$ and $\ell=1$. The introduction of operator $s_{j}^{z}$ imposes the evaluation of the trace of the operator $s_{j}^{z} \exp \left(-\beta H_{\jmath}^{\text {ex }}\right)$. In a first step we express the operator $s_{j}^{z}$ on the orthogonal basis $\left(\mathbf{i}_{j}^{\prime}, \mathbf{j}_{j}^{\prime}, \mathbf{k}^{\prime}{ }_{j}\right)$ in which $\mathbf{k}^{\prime}$ is orientated along the direction of vector $\mathcal{S}_{J}$ (cf. Eq. (2)); let $\tilde{s}_{j}^{x}, \tilde{s}_{j}^{y}$ and $\tilde{s}_{j}^{z}$ be the new operators; noting that $\tilde{s}_{j}^{x}$ and $\tilde{s}_{j}^{y}$ have no diagonal elements and owing to equation (2) the calculation of this trace reduces to

$$
\operatorname{Tr}_{\mathbf{s}_{\jmath}}\left(s_{\jmath}^{z} \exp \left(-\beta J \mathcal{S}_{\jmath} \cdot \mathbf{s}_{\jmath}\right)\right)=\mathbf{k}_{\jmath} \mathbf{k}_{j}^{\prime} \operatorname{Tr}_{\mathbf{s}_{\jmath}}\left(\tilde{s}_{\jmath}^{z} \exp \left(-\beta J \mathcal{S}_{\jmath} \cdot \tilde{s}_{\jmath}^{z}\right)\right)
$$

with

$$
\mathbf{k}_{\jmath} \quad \mathbf{k}_{\jmath}^{\prime}=\frac{(1+\alpha) S_{3}^{z}+(1-\alpha) S_{3+1}^{z}}{\sqrt{2\left(1+\alpha^{2}+\left(1-\alpha^{2}\right) \cos \Theta_{2, j+1}\right)}} .
$$

Then expanding $S_{\jmath}^{z}$ and $S_{\jmath+1}^{z}$ with equation (14) we have

$$
\begin{gathered}
\operatorname{Tr}_{\mathbf{s}_{\mathfrak{j}}}\left(s_{\mathrm{j}}^{z} \exp \left(-\beta J \mathcal{S}_{\mathbf{j}} \cdot \mathbf{s}_{\mathbf{j}}\right)\right)=-\beta J \sqrt{\frac{4 \pi}{3}}\left[(1+\alpha) Y_{1}^{0}\left(\mathbf{S}_{\jmath}\right)+(1-\alpha) Y_{1}^{0}\left(\mathbf{S}_{\jmath+1}\right)\right] \\
\times \sum_{\sigma=-s}^{+s} \sigma \frac{\exp \left(\sigma \lambda \sqrt{1+\eta \cos \Theta_{\jmath, \jmath+1}}\right)}{\lambda \sqrt{1+\eta \cos \Theta_{\jmath, \jmath+1}}} .
\end{gathered}
$$

In this expression the summation over $\sigma$ only depends on the angle $\Theta_{\jmath, \jmath+1}$ between vectors $\mathbf{S}_{3}$ and $\mathbf{S}_{3+1}$. Therefore, as for equation (7), it can be expanded on the infinite basis of spherical harmonics. We obtain an expression similar to that given by equation (9) in which 
the coefficient $A_{\ell}(\lambda, \eta, s)$ is now replaced by a coefficient $B_{\ell}(\lambda, \eta, s)$ defined as follows

$$
B_{\ell}(\lambda, \eta, s)=2 \pi \sum_{\sigma=-s}^{+s} \sigma^{2} \int_{-1}^{+1} \frac{\exp (\sigma \lambda \sqrt{1+\eta x})}{\sigma \lambda \sqrt{1+\eta x}} P_{\ell}(x) \mathrm{d} x .
$$

Then, the orthogonality condition and the specific properties of spherical harmonics allow one to select the values $m=0$ and $\ell=1$. Finally we have

$$
\begin{gathered}
\left\langle S_{\imath}^{z} S_{\jmath}^{z}>=P^{\jmath-\imath},<S_{\imath}^{z} s_{\jmath}^{z}>=Q_{1} P^{\jmath-\imath}\right. \\
<s_{\imath}^{z} S_{\jmath}^{z}>=Q_{-1} P^{\jmath-\imath-1},<s_{\imath}^{z} s_{\jmath}^{z}>=Q_{1} Q_{-1} P^{\jmath-\imath-1},
\end{gathered}
$$

where

$$
\begin{gathered}
P=\frac{A_{1}(\lambda, \eta, s)}{A_{0}(\lambda, \eta, s)}, \\
Q_{\varepsilon}=-\beta J \frac{(1+\varepsilon \alpha) B_{0}(\lambda, \eta, s)+(1-\varepsilon \alpha) B_{1}(\lambda, \eta, s)}{A_{0}(\lambda, \eta, s)}, \varepsilon= \pm 1
\end{gathered}
$$

with

$$
\begin{gathered}
A_{1}(\lambda, \eta, s)=\frac{4 \pi}{\left(\lambda^{2} \eta\right)^{2}} \sum_{\sigma=-s}^{+s} \sum_{\varepsilon= \pm 1} \frac{\varepsilon}{\sigma^{4}}\left(v_{\sigma, \varepsilon}^{3}-3 v_{\sigma, \varepsilon}^{2}+\left(6-\sigma^{2} \lambda^{2}\right)\left(v_{\sigma, \varepsilon}-1\right)\right) \\
\times \exp \left(v_{\sigma, \varepsilon}\right) \\
B_{0}(\lambda, \eta, s)=\frac{4 \pi}{\lambda^{2} \eta} \sum_{\sigma=-s}^{+s} \sum_{\varepsilon= \pm 1} \varepsilon \exp \left(v_{\sigma, \varepsilon}\right) \\
B_{1}(\lambda, \eta, s)=\frac{4 \pi}{\left(\lambda^{2} \eta\right)^{2}} \sum_{\sigma=-s}^{+s} \sum_{\varepsilon= \pm 1} \frac{\varepsilon}{\sigma^{2}}\left(v_{\sigma, \varepsilon}^{2}-2 v_{\sigma, \varepsilon}+2-\sigma^{2} \lambda^{2}\right) \exp \left(v_{\sigma, \varepsilon}\right)
\end{gathered}
$$

In these previous expressions, $\lambda, \eta, A_{0}(\lambda, \eta, s)$ and $v_{\sigma, \varepsilon}$ are given by equations (8) and (12), respectively. Then using the expressions of the varıous spin-spin correlations in the susceptibility definition (cf. Eq. (13)) we have for a unit cell

$$
\chi=\frac{\beta(g s)^{2}}{3}\left[r^{2}+\frac{s+1}{s}+2 \frac{r^{2} P+r\left[\left(Q_{1}+Q_{-1}\right) / s\right]+\left(Q_{1} Q_{-1} / s^{2}\right)}{1-P}\right],
$$

where $r$ is the ratio of the magnetic moment magnitudes per unit cell

$$
r=\frac{G}{g s} .
$$

Finally it must be noticed that the evaluation of the susceptibility just necessitates the knowledge of the four coefficients $A_{0}, A_{1}, B_{0}$ and $B_{1}$ previously defined, whatever the value of the spin quantum number $s$. Note also that all these coefficients can be easily calculated in 
the classical-spin approximation by assuming the following transformation

$$
\frac{1}{2 s+1} \sum_{\sigma=-s}^{+s} f\left(\frac{\sigma}{s}\right)=\int_{-1}^{+1} f(x) \mathrm{d} x, \quad \text { as } s \rightarrow+\infty .
$$

However, when $s$ becomes infinite, in order to keep a physical meaning, the quantum spin moment magnitude $g s$ as well as the exchange energy $J s$ are constrained to remain finite through a convenient adaptation of $g$ and $J$, respectively.

Another parameter of significant importance, in the present context, is the correlation length defined as follows

$$
\xi=\left(\frac{1}{2} \frac{\sum_{n=0}^{+\infty} n^{2}\left|<S_{2}^{z} S_{\imath+n}^{z}>\right|}{\sum_{n=0}^{+\infty}\left|<S_{\imath}^{z} S_{\imath+n}^{z}>\right|}\right)^{1 / 2}
$$

Then, taking into account the expression of $\left\langle S_{\imath}^{z} S_{\imath+n}^{z}\right\rangle$ (cf. Eq. (19)) it is easily shown that

$$
\xi=\left(\frac{1}{2} \frac{|P|(1+|P|)}{(1-|P|)^{2}}\right)^{1 / 2}
$$

\section{Study of the Low-Temperature Behavior}

The low-temperature study is mainly conditioned by the classification of the parameter $\alpha$ with respect to unity. When $\alpha<1$, the exchange interactions $J(1+\alpha)$ and $J(1-\alpha)$, involved per unit cell, have the same sign (i.e. that of $J$ ); if $J<0$ all the couplings are ferromagnetic and, at $0 \mathrm{~K}$, all the spin moments are oriented towards the same direction (see Fig. 2a); on the contrary, if $J>0$ all the couplings are antiferromagnetic and, at $0 \mathrm{~K}$, two consecutive spin moments are oriented along opposite directions (see Fig. 2b). When $\alpha>1$, the involved exchange interactions $J(1+\alpha)$ and $J(1-\alpha)$ now have opposite signs (i.e. that of $J$ and $-J$ ); in other words we have a regular alternation of ferromagnetic and antiferromagnetic couplings and the chain shows a vanishing global moment at $0 \mathrm{~K}$ (see Fig. 2c). Note that, when $\alpha=1$, we deal with a dimerized chain.

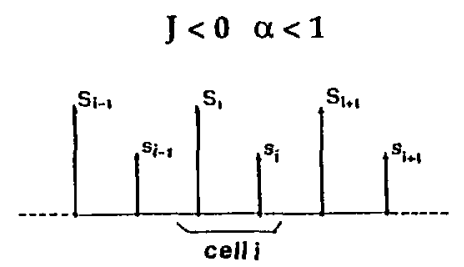

a

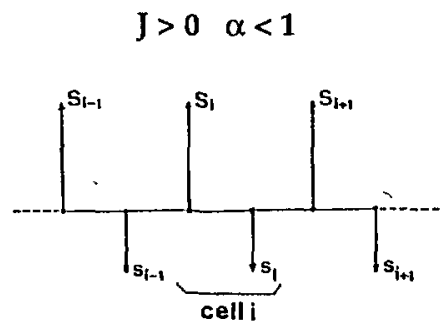

b

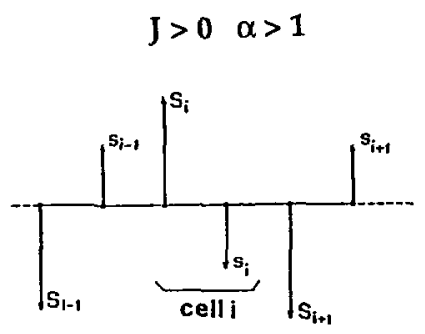

c

Fig. 2. - Structures of the chain ground states for $\alpha<1$ (Fig. 2a: $J<0$; Fig. 2b: $J>0$ ) and for $\alpha>1$ (Fig. 2c: $J>0$ ). 
The low-temperature behavior study of the susceptibility necessitates the evaluation of quantities $P, Q_{1}$ and $Q_{-1}$ in that range of temperatures; more exactly, because of their respective definitions, their behavior is given by that of the ratios $A_{1} / A_{0}, B_{1} / A_{0}$ and $B_{0} / A_{0}$ which have been introduced in the previous section (cf. Eqs. (12), (21)-(23)). As these coefficients contain exponential terms, their low-temperature behavior is mainly given by these factors character1zed by the maximum argument $2 \beta|J| s$ (note that, as two consecutive arguments only differ by the quantity $-2 \beta|J|$, this approximation remains valid when the temperature is plainly lower than $|J|)$. All the main results of interest have been summarized in Table I.

Whatever the value of $\alpha$ with respect to unity, in the low-temperature range, $|P|$ tends to unity according to a $T$-law. Consequently, the examination of equation (28) allows one to say that the correlation length $\xi$ behaves as $(1-|P|)^{-1}$ and thus diverges according to a $T^{-1}$-law (see Tab. I). This aspect has been previously encountered $[19,33,36,38]$ and appears as the signature of isotropıc couplings involving classical spin moments alternating with quantum ones (or different classical ones). Note that, when $\alpha=1$ (case of dimerized chains), $P$ vanishes and the correlation length has no more physical interest. Thus the low-temperature behavior of the susceptibility is mainly given by that of $(1-P)^{-1}$ on condition that the numerator of the fraction containing the term $1-P$ (cf. Eq. (24)) does not vanısh; at this step it must be noted that, in the low-temperature domain, this numerator is neither more nor less than the square of the magnetic moment $M$ per unit cell. In other words the low-temperature behavior of $\chi T$ is mainly given by that of $\xi M^{2}$. Therefore the chain can be considered as an assembly of quasi-rigid quası-independent blocks, each one of length $\xi$ and moment $M$ per unit cell.

Table I. - Low-temperature behavior of the susceptibility and the correlative quantities of main interest for various significant values of the exchange parameter $\alpha$.

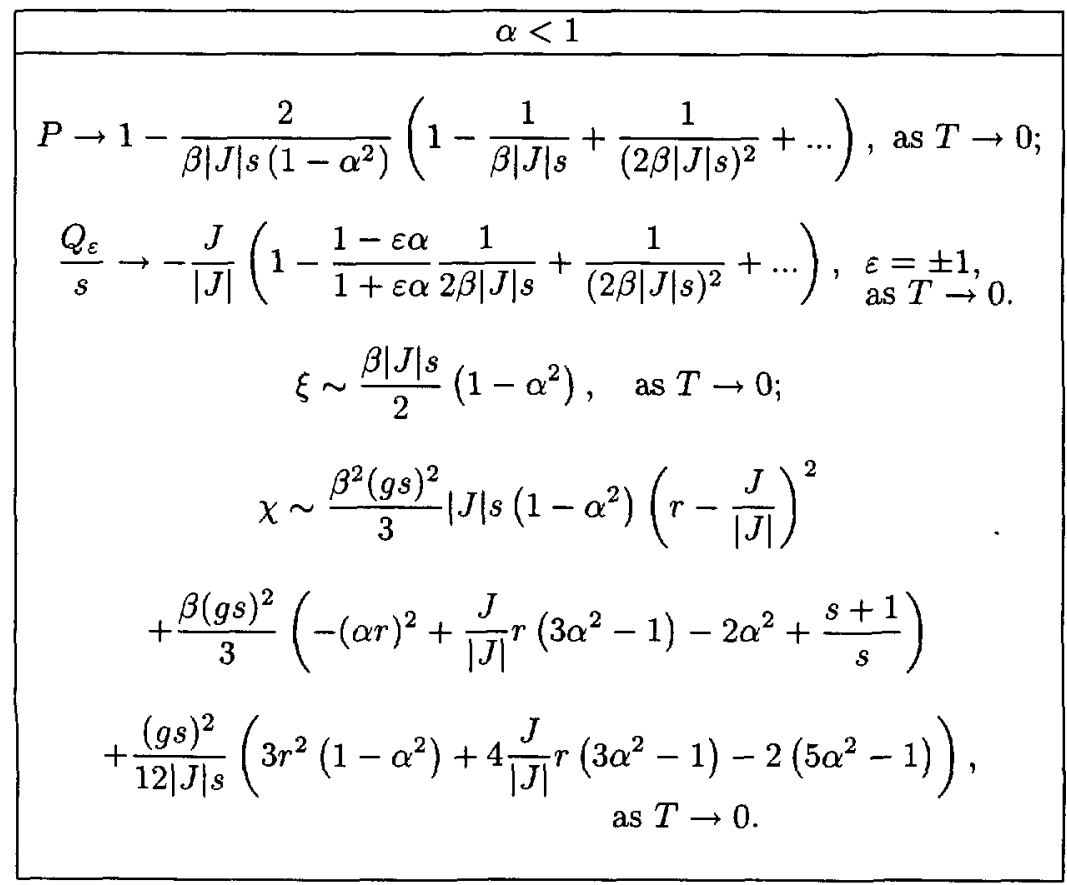


Table I. - (continued)

$$
\begin{aligned}
& \begin{array}{c}
\alpha=1 \\
P=0 \quad \forall T ; \\
\frac{Q_{\varepsilon}}{s} \rightarrow-\frac{J}{|J|} \frac{1+\varepsilon}{2}, \varepsilon= \pm 1, \text { as } T \rightarrow 0 . \\
\xi=0 \quad \forall T ; \\
\chi \sim \frac{\beta(g s)^{2}}{3}\left(r^{2}+\frac{s+1}{s}-2 \frac{J}{|J|} r\right), \text { as } T \rightarrow 0 .
\end{array} \\
& \begin{array}{c}
\alpha>1 \\
P \rightarrow-\left(1-\frac{2 \alpha}{\alpha^{2}-1} \frac{1}{\beta|J| s}+\ldots\right), \text { as } T \rightarrow 0 \\
\frac{Q_{\varepsilon}}{s} \rightarrow-\varepsilon \frac{J}{|J|}\left(1+\frac{1-\varepsilon \alpha}{1+\varepsilon \alpha} \frac{1}{2 \alpha \beta|J| s}+\ldots\right), \varepsilon= \pm 1, \text { as } T \rightarrow 0 . \\
\xi \sim \frac{\beta|J| s}{2 \alpha}\left(\alpha^{2}-1\right), \text { as } T \rightarrow 0 \\
\chi \sim \frac{\beta(g s)^{2}}{3 s}+\frac{(g s)^{2}\left(\alpha r-\frac{J}{|J|}\right)^{2}}{3 \alpha\left(\alpha^{2}-1\right)|J| s}, \text { as } T \rightarrow 0
\end{array}
\end{aligned}
$$

When $\alpha<1$, all the couplings are ferromagnetic $(J<0)$ or antiferromagnetic $(J>0)$; for $r \neq 1$ the chain shows a global moment. If we examine the general low-temperature expansion of the susceptibility given in Table I, the first term appears to be the dominant one; thus the product $\chi T$ behaves as

$$
\chi T \sim \frac{\beta(g s)^{2}}{3}|J| s\left(1-\alpha^{2}\right)\left(r-\frac{J}{|J|}\right)^{2}, \quad \text { as } T \rightarrow 0, \quad \alpha<1, \quad r \neq 1 .
$$

This behavior is illustrated by the curves of Figure 3 where we have considered the value $\alpha=0.50$; as expected the divergence law is enhanced if couplings are ferromagnetic (the factor $r-1$ is replaced by $r+1)$. For antiferromagnetic couplings, in the compensation case $(r=1)$, the chain has no net moment at $0 \mathrm{~K}$ : The behavior of $\chi T$ is given by the competition between the divergence of the correlation length $\xi\left(T^{-1}\right.$-law) and the evanescence of $M$, the magnetic moment per unit cell ( $T$-law). As the factor $r-J /|J|$ involved in equation (29) vanishes, it is necessary to consider further terms of the low-temperature expansion of the susceptibility (Tab. I). Then, under this condition, it appears that the product $\chi T$ shows a constant limit 


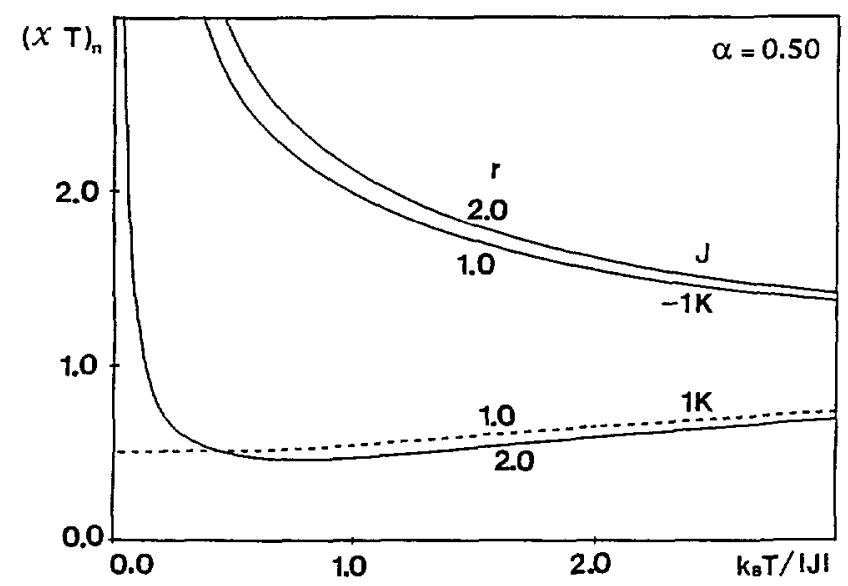

Fig. 3. - Thermal variations of the product $(\chi T)_{n}$ for a $(\mathrm{S}, 1 / 2)_{N}$ chain showing isotropic couplings for several values of $r$ and $J(g=2, \alpha=0.50)$.

$$
\chi T \rightarrow \frac{(g s)^{2}}{3 k_{\mathrm{B}} s}+\frac{(g s)^{2}\left(1-\alpha^{2}\right)}{12|J| s} T+. ., \quad \text { as } T \rightarrow 0, \quad \alpha<1, \quad r=1 .
$$

At this step it must be also noted that the $T$-vanishing law of $M$ is characteristic of isotropic coupled spin chains showing an alternation of classical and quantum spin moments (or different classical ones). This behavior which recalls a Curie law has no common point with the paramagnetic behavior of free moments. In particular the possibility of confusion with paramagnetic impurities must not been ignored when one tries to interpret experımental data. Practically, the rigorous moment compensation $(r=1)$ is seldom encountered but the examination of the curves of Figure 4 allows one to say that, for observing a very different behavior with respect to the Curie law, it is necessary to consider temperatures closer and closer to $0 \mathrm{~K}$ if the ratio $r$ of

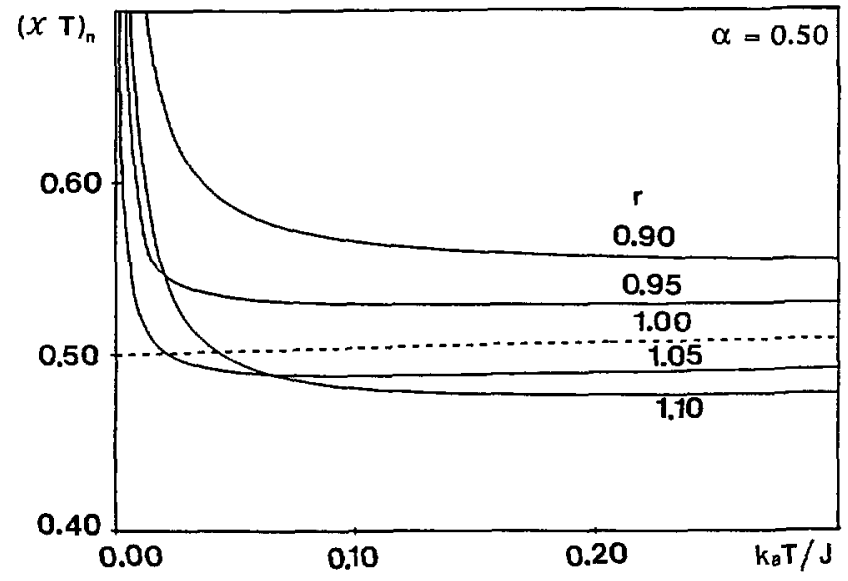

Fig. 4. - Thermal variations of the product $(\chi T)_{n}$ for a $(\mathrm{S}, 1 / 2)_{N}$ chain showing isotropic couplings for several values of $r(J>0, g=2, \alpha=0.50)$. 
the magnetic moment magnitudes is closer to unity. When $\alpha>1$, the couplings with nearest neighbors are alternatively ferromagnetic and antiferromagnetic. Whatever the sign of $J$, the chain ground state at $0 \mathrm{~K}$ is characterized by a vanishing moment. This case appears to be very similar to the previous one where $\alpha<1, J>0$ and $r=1$. Thus, in the low-temperature range, we hàve

$$
\chi T \rightarrow \frac{(g s)^{2}}{3 k_{\mathrm{B}} s}+\frac{(g s)^{2}\left(\alpha r-\frac{J}{|J|}\right)^{2}}{3 \alpha\left(\alpha^{2}-1\right)|J| s} T+. ., \quad \text { as } T \rightarrow 0, \quad \alpha>1 .
$$

Therefore, the product $\chi T$ follows a Curie law in spite of the absence of free magnetic moments and whatever the sign of $J$. Moreover the $T$-vanishing law of $\chi T$ is due to the fact that, in the low-temperature domain, $\chi T$ precisely behaves as $\xi M^{2}$, where $\xi$ diverges as $T^{-1}$ (see Tab. I) and $M$ vanishes as $T$. However, in contrast with the case $\alpha<1$, the ratio $r$ has no major effect. It must be only noticed that the initial slope of the thermal variation of $\chi^{T}$ vanishes without any change of sign if $r=1 / \alpha$. All these aspects are illustrated by the curves of Figure 5 where we have considered the value $\alpha=1.50$. At this step it is of great interest to detail the deep reasons which are responsible for such a behavior because they involve a more subtle mechanısm. By using the low-temperature behavior of $P, Q_{1}$ and $Q_{-1}$ (see Tab. I) in equation (24) it is easily shown that $(1-P)^{-1}$ no longer diverges; in addition, if we consider all the correlations concerning the different classical moments, they exactly compensate the classical self correlation. But, for the quantum moments, the self correlation $s(s+1)$ is not exactly compensated by all the correlations involving different quantum spin moments $\left(-s^{2}\right)$. Therefore we deal with a purely quantum effect, the importance of which weakens when $s$ becomes infinite (i.e. if we tend to the classical spin approximation).

Nevertheless it remains to determine if the classical moments (which alternate with the

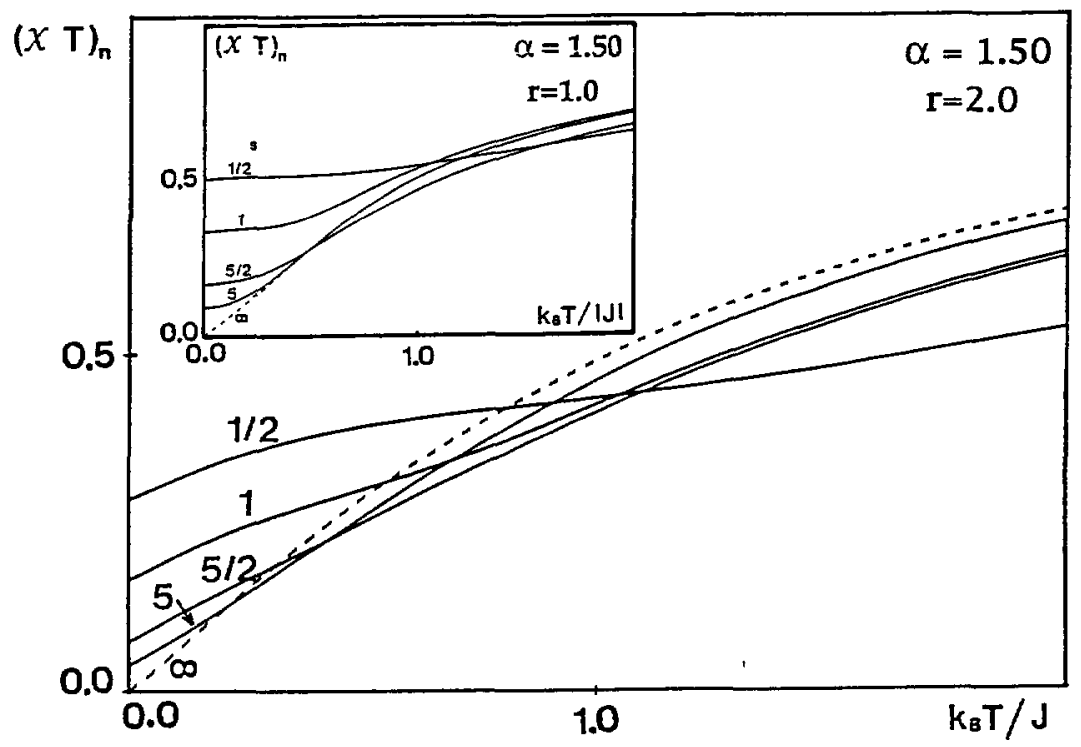

Fig. 5. - Thermal varıations of the product $(\chi T)_{n}$ for a $(\mathbf{S}, \mathbf{s})_{N}$ chain showing isotropic couplings for several values of $s(J>0, g s=1, \alpha=1.50, r=2.0)$; the dashed line corresponds to the value of $(\chi T)_{n}$ in the classical spin approximation (inset: $J>0, g s=1, \alpha=1.50, r=1.0$ ). 
quantum ones) play an important role in this effect. For examining this specific point, we have achieved the exact numerical calculation of the properties of a tetramer made up of four quantum spin moments (see inset of Fig. 6) [36]; the exchange term is described by equation (2) in which $\mathbf{S}_{2}$ and $\mathbf{S}_{2+1}$ are here replaced by $\mathbf{s}_{1}$ and $\mathbf{s}_{2}$ (respectively $\mathbf{s}_{2}$ and $\mathbf{s}_{1}$ ) if $\mathbf{s}_{2}$ is $\mathbf{s}^{\prime}{ }_{1}$ (respectively $\mathbf{s}_{2}{ }_{2}$ ). As the exchange energy $J$ is positive and $\alpha>1$, one can say that the coupling is ferromagnetic for pairs $\left(\mathbf{s}_{1}, \mathbf{s}_{2}^{\prime}\right)$ and $\left(\mathbf{s}_{2}, \mathbf{s}^{\prime}{ }_{1}\right)$, and antiferromagnetic for pairs $\left(\mathbf{s}_{1}, \mathbf{s}_{1}^{\prime}\right)$ and $\left(\mathbf{s}_{2}, \mathbf{s}_{2}{ }_{2}\right)$. The spin quantum numbers are such as $s=1 / 2$ for $\mathbf{s}_{1}$ and $\mathbf{s}_{2} ; s^{\prime}{ }_{1}$ and $s_{2}^{\prime}$ are characterized by $s^{\prime}$ (when $s^{\prime}$ becomes infinite - classical spin approximation - this numerical work has been adapted conveniently). In Figure 6 we have reported the thermal variations of $\chi_{n}$ for various values of $s^{\prime}$ and for $\alpha=1.50$ and $r=2.0$; note that the Landé factors as well as the exchange energy $J$ have been adapted so that $g^{\prime} s^{\prime}$ and $J s^{\prime}$ respectively remain unchanged when s' is modified. Thus we deal with a short cyclic chain the characteristics of which are very close to that of the previous $(\mathbf{S}, 1 / 2)_{N}$ spin chain when $s^{\prime}$ becomes infinite. The tetramer ground state is a singlet one but, when the value of $s^{\prime}$ increases, the density of multiplet states increases in the close neighborhood of the ground state. Under these conditions, the susceptibility shows a more and more narrow maximum for a temperature which is closer and closer to $0 \mathrm{~K}$, as $s^{\prime}$ increases; then $\chi_{n}$ rapidly decreases to a constant value, at $0 \mathrm{~K}$, which corresponds to the Van Vleck contribution. When $s^{\prime}$ becomes infinite (classical spin approximation) $\chi$ is maximum at $0 \mathrm{~K}$. This can be directly observed on the curves of Figure 6 . Under addition, one can notice that the non-vanishing value of $\chi$ increases when the ratio $s^{\prime} / s$ strongly deviates from unity. Therefore, for the infinite spin chain, the divergence of $\chi$ can be interpreted as an enhancement of the Van Vleck contribution to the susceptibility, due to the fact that the quantum spin coherence occurs at lengths which become infinite. Thus, though we have considered a finite length chain, this low temperature study confirms that the classical character of spin moments alternating with quantum ones can deeply modify the susceptibility behavior.

Now we briefly recall below an experimental illustration previously published which has intiated this theoretical model [19]. It concerns the interpretation of the magnetic properties of the compound $\mathrm{MnCu}(\mathrm{obp})\left(\mathrm{H}_{2} \mathrm{O}\right)_{3} \cdot \mathrm{H}_{2} \mathrm{O}$ where obp is an abbreviation for the ligand oxa-

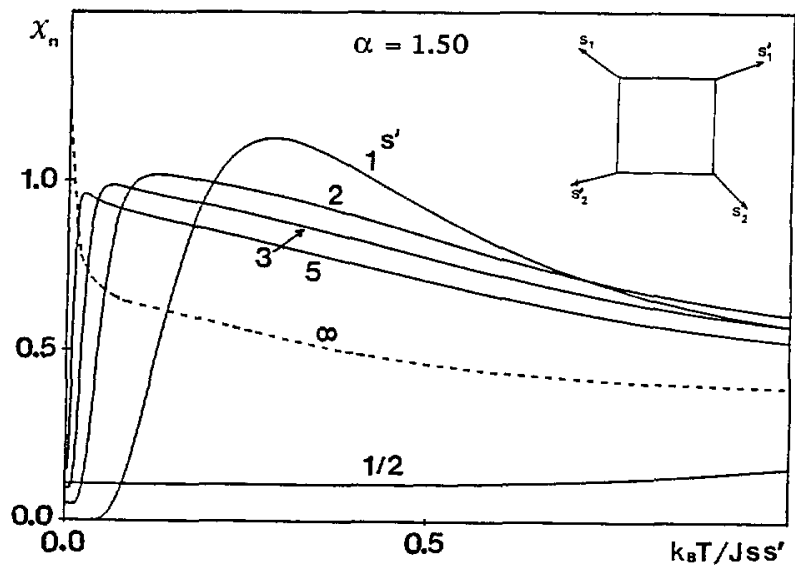

Fig. 6. - Thermal varıations of the susceptibility $\chi_{n}$ for a $\left(\mathbf{s}^{\prime}, 1 / 2\right)$ tetramer showing isotropic couplings for several values of $s^{\prime}\left(J>0, g=2, g^{\prime} s^{\prime}=2, \alpha=1.50, r=2.0\right)$; the dashed line corresponds to the value of $\chi_{n}$ in the classical spin approximation (inset: tetramer structure). 

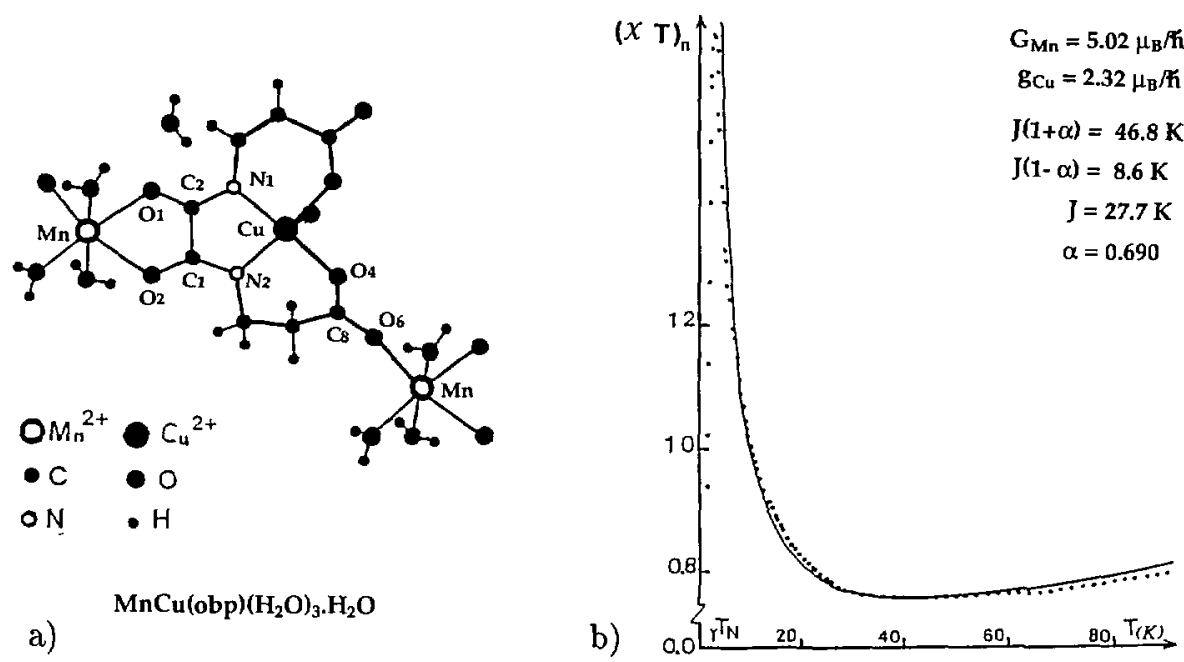

Fig. 7. - Structure of the ferrmagnetic chains in the compound $\mathrm{MnCu}(\mathrm{obp})\left(\mathrm{H}_{2} \mathrm{O}\right)_{3} \cdot \mathrm{H}_{2} \mathrm{O}(\mathrm{Fig} .7 \mathrm{a}$ from Ref. [19]); experımental results (dotted line) and theoretical curve for the thermal varıations of the product $(\chi T)_{n}$ for a powder of $\mathrm{MnCu}(\mathrm{obp})(\mathrm{H} 2 \mathrm{O})_{3} \cdot \mathrm{H}_{2} \mathrm{O}$ (Fig. $7 \mathrm{~b}$ from Ref. [37]).

midobis(propionato). The structure of this compound is shown in Figure 7a. It can be described with the help of well-isolated chains (from a magnetic point of view) characterized by the alternation of cations $\mathrm{Mn}^{2+}$

$\left(s^{\prime}=5 / 2\right)$ and $\mathrm{Cu}^{2+}(s=1 / 2)$; note that, as soon as a spin quantum number reaches the value $5 / 2$, the classical spin approximation is usually employed; consequently, one can say that the present compound is characterized by $(S, 1 / 2)_{N}$ chains. Within the chain, the Mn and $\mathrm{Cu}$ atoms are bridged by an oxamido group $\mathrm{O}_{1}-\mathrm{O}_{2}-\mathrm{C}_{1}-\mathrm{C}_{2}-\mathrm{N}_{1}-\mathrm{N}_{2}$ with a $\mathrm{Mn}$-Cu separation of $5.452 \AA$ and by a carboxylato group $\mathrm{O}_{4}-\mathrm{C}_{8}-\mathrm{O}_{6}$ with a $\mathrm{Mn}-\mathrm{Cu}$ separation of $6.066 \AA$. Two distinct exchange parameters are expected; moreover the nature of host sites, on the one hand, and the involved ions, on the other hand, allow one to consider that the interactions between nearest neighbors are of the Heisenberg type. The best fitting of susceptibility measurements (see Fig. 7b) is obtained for the couple of values $J(1+\alpha)=46.8 \mathrm{~K}$ and $J(1-\alpha)=8.6 \mathrm{~K}$, thus leading to $\alpha=0.69$ and $J=27.7 \mathrm{~K}$. It is reasonable to attribute the strong value of the exchange energy to the oxamido bridge $[7,14,15,17]$. It is remarkable that the value of the exchange energy between $\mathrm{Mn}^{2+}$ and $\mathrm{Cu}^{2+}$ has already been determined in structures for which these ions, considered in the same host sites, are bridged by oxamato $(36 \mathrm{~K})$ and oxalato $(26 \mathrm{~K})$ groups, deduced from oxamido by respectively substituting one or both NR groups by oxygen atoms $[7,17,39,40]$. Therefore, these values are in perfect agreement with that precisely obtained for the oxamido group $(46.8 \mathrm{~K}$ ) if we consider that there is a simple linear variation of the exchange energy with respect to the number of oxygen atoms involved in the bridge between $\mathrm{Mn}^{2+}$ and $\mathrm{Cu}^{2+}$ 


\section{Conclusion}

In the present work we have set a general formulation for solving the statistical problem of a very large class of one-dimensional ferrimagnets made up of two sublattices $(S, s)_{N}$ and characterized by isotropic couplings between nearest neighbors as well as two exchange parameters. We have shown that it is only possible to derive simple closed-form expressions of the zero-field partition function, the spin-spin correlations as well as for the susceptibility. We have examined the cases where the chain is exclusively composed of ferromagnetic or antiferromagnetic couplings, or shows a regular alternation of these two types of couplings. In all cases, in the lowtemperature range, the chain can be described as an assembly of quasi-independent quasi-rigid blocks, each one of length $\xi$, the correlation length, and moment $M$, the magnetic moment per unit cell. Therefore the product $\chi T$ behaves as $\xi M^{2}$; in particular, in the magnetic moment compensation, its behavior is mainly described by the competition between the divergence of $\xi$ and the evanescence of $M$. This has allowed us to show subtle aspects of both short- and long-range orderings. Finally we have recalled the interpretation of the experimental results obtained on the compound $\mathrm{MnCu}(\mathrm{obp})\left(\mathrm{H}_{2} \mathrm{O}\right)_{3} \cdot \mathrm{H}_{2} \mathrm{O}$ [where obp=oxamidobis(propionato)] because it has initiated the present theoretical work. The problem of isotropic chains showing randomly distributed parameters has not been examined; the present model can be easily generalized by taking into account these aspects. So far we have just considered the case of exchange couplings randomly distributed [19]; but, from an expermmental point of view, the important problem of cationic vacancies can also be considered in a wider generalization of the present work. Therefore we believe that this theoretical work can be of great help for future experimental data discussion and the further investigations of interesting one-dimensional compounds.

\section{References}

[1] The Physics and Chemistry of Low Dimensional Solids, L. Alcacer, Ed. (Reidel, Dordrecht, 1980).

[2] Physics in One Dimension, J. Bernasconi and T. Schneider, Eds. (Sprınger-Verlag, Berlın, 1981), Vol. 23.

[3] Organıc and Inorganic Low-Dimensıonal Crystalline Materıals, P. Delhaes and M. Drillon, Eds. (Plenum, New-York, 1987) Vol.B 168.

[4] de Jongh L.J. and Miedema A.R., Adv. Phys. 23 (1974) 1.

[5] Verdaguer M., Gleizes A., Renard J.P. and Sletten J., Phys. Rev. B29 (1984) 5144.

[6] Drillon M., Coronado E., Beltran D., Curély J., Georges R., Nugteren P.R., de Jongh L.J. and Genıcon J.L., J. Magn. Magn. Mater. 54-57 (1986) 1507.

[7] Pei Y., Verdaguer M., Kahn O., Sletten J. and Renard J.P., Inorg. Chem. 26 (1987) 138.

[8] Hatfield W.E., Estes W.E., Marsh W.E., Pickens M.W., Ten Haar L.W. and Weller R.W., Extended Linear Chain Compounds, J.S. Miller, Ed. (Plenum, New-York, 1983) Vol. 3, p. 43.

[9] Willet R.D., Gaura R.M. and Landee C.P., Extended Linear Chain Compounds, J.S. Miller, Ed. (Plenum, New-York, 1983) Vol. 3, p. 143.

[10] Renard J.P., Clément S. and Verdaguer M., Proc. Indian Acad. Sci., Chem. Sci. 98 (1987) 131.

[11] Olmstead M.M., Musker W.K., Ten Haar L.W. and Hatfield W.E., J. Am. Chem. Soc. 104 (1982) 6627.

[12] Wolthuis A.J., Huiskamp W.J., de Jongh L.J. and Reedijk J., Physıca $B+C$ 133B+C (1985) 161. 
[13] Daoud A., Ben Salah A., Chappert C., Renard J.P., Cheikh-Rouhou A., Duc T. and Verdaguer M., Phys. Rev. B33 (1986) 6253.

[14] Gleizes A. and Verdaguer M., J. Am. Chem. Soc. 103 (1981) 7373.

[15] Gleizes A. and Verdaguer M., J. Am. Chem. Soc. 106 (1984) 3727.

[16] Beltran D., Escriva E. and Drillon M., J. Chem. Soc., Faraday Trans. II 78 (1987) 1773.

[17] Pei Y., Sletten J. and Kahn O., J. Am. Chem. Soc. 108 (1986) 3143.

[18] Pei Y., Kahn O., Sletten J., Renard J.P., Georges R., Curély J., Gianduzzo J.C. and Xu Q., Inorg. Chem. 27 (1988) 47.

[19] Georges R., Curély J., Gianduzzo J.C., Xu Q., Kahn O. and Pei Y., Physica 153B (1988) 77.

[20] Georges R. and Kahn O., Mol. Cryst. Liq. Cryst. 176 (1989) 473.

[21] Sapina F., Coronado E., Drillon M., Georges R. and Beltran D., J. Phys. France 49 (1988) C8-1423.

[22] Georges R. and Curély J., J. Chem. Phys. 86 (1989) 967.

[23] Orbach R., Phys, Rev. 112 (1958) 309.

[24] Rushbrooke G.S., Baker G.A. and Wood P.J., Phase Transition and Critical Phenomena, C. Domb and M.S. Green, Eds. (Academic, New York, 1974) Vol. 3.

[25] Baker G.A., Gilbert H.E., Eve J. and Rushbrooke G.S., Phys. Rev. 164 (1974) 800.

[26] Domb C., Adv. Phys. 19 (1970) 339.

[27] Kondo J. and Yamaji K., Prog. Theor. Phys. 47 (1972) 807.

[28] Bonner J.C. and Fisher M.E., Phys. Rev. 135 (1964) 640.

[29] Blöte H.W., Physica 79B (1975) 427.

[30] de Neef T., Phys. Rev. B13 (1976) 4141.

[31] Drillon M., Gianduzzo J.C. and Georges R., Phys. Lett. 96A (1983) 413.

[32] Drillon M., Coronado E., Georges R., Gianduzzo J.C. and Curély J., Phys. Rev. B 40 (1989) 10992.

[33] Fisher M.E., Am. J. Phys. 32 (1964) 343.

[34] Stanley H.E., Phys. Rev. 179 (1969) 570.

[35] Seiden J., J. Phys. Lett. 44 (1983) L947.

[36] Curély J., Thèse de Doctorat, Université de Bordeaux I (1990).

[37] Curély J., Georges R., Gianduzzo J.C., Xu Q., Kahn O., Pei Y. and Sletten J., Organıc and Inorganic Low-Dimensıonal Crystalline Materıals, P. Delhaes and M. Drillon, Eds. (Plenum, NewYork, 1987), Vol. B 168, p. 413.

[38] Curély J. and Georges R., Phys, Rev. B46 (1992) 3520.

[39] Pei Y., Verdaguer M., Kahn O., Sletten J. and Renard J.P., J. Am. Chem. Soc. 108 (1986) 7428.

[40] Verdaguer M., Julve M., Michalowicz A. and Kahn O., Inorg. Chem. 22 (1983) 2624. 